\title{
Introduction to A Research Agenda for Global Rural Development
}

\section{Setting some seeds for the research agenda in rural social science}

For our contribution to the Elgar Research Agendas series, we three longstanding rural and environmental researchers have come together to begin to answer the timely and creative question of, what is the current research agenda for rural development studies? This is clearly an ambitious and challenging question too. As authors, we have been pursuing internationally comparative research over most of our long careers, as well as being based deeply within our own national research cultures in France, the UK and Brazil. We have also increasingly evolved as interdisciplinary rural researchers, as indeed the sub-disciplines of rural sociology, geography and development, and rural social science have merged not least as a result of the wider challenges of the environmentalization of social science (see Marsden (ed) 2018). Moreover, this is not unrelated to the radical questions being posed about global rural development in a post-colonial, increasingly globalized and 'underdeveloping' as well as 'developing' world. There are clearly many routes to rural 'development' and clearly competing ideas and theorizations of modernities.

Indeed, the whole question of rural 'development', it can be argued, is now in need of critical 'surgery' in a non-deterministic, open-ended and post-disciplinary way. As authors, we are strongly rooted in and associated with the traditional disciplines of rural sociology and rural geography, but this book, in both its critical reviews of the 'state of the art' and its suggestions for the future research agenda, adopts a wider canvas. This is to contribute to how research both on and with rural peoples in their places and spaces needs to be directed and practiced in (as we start in Chapter 1) an increasingly urban, 
resource intensive and cosmopolitan world; where mobilities, sustainabilities and a host of vulnerabilities continue to collide. Without doubt, the study of the rural can no longer be seen as the study of stable, traditional communities, if it ever could. Rather, we propose to apply a critical conceptual mirror to the rural, examining its re-location and re-positioning at the heart of global problems and increasingly cosmopolitan cultures. Ruralities and the rural experience become more significant in a globalized and cosmopolitan world. This necessitates a post-disciplinary approach, one that, for instance, blends disciplines like anthropology, environmental politics, heterodox economics and environmental philosophy into its vectors of scholarly endeavour and investigation.

\section{A working conceptual model for global rural development: the emerging rural development agenda}

This volume explores and develops key areas and themes of the rural development research agenda by focussing upon eight key dimensions (see Figure 0.1): new ruralities, governance, power and transformation, financialization, land and bio-sphere rights, agroecology, family farming, and markets and exchange. We have prioritized these areas for several reasons. First, and overall, they clearly exemplify and analytically and conceptually develop all eight of the more generic and cross-cutting themes outlined below. Second, we argue that in a contemporary sense the eight key dimensions represent critical areas for the research agenda for the increasingly interdisciplinary and transdisciplinary areas of rural development. And third, the chapters all combine a retrospective, contemporary and prospective lens to different bodies of international research and research practice, which are of critical concern. In each of the chapters we also attempt to provide an internationally comparative perspective, by citing empirical work from our own both individual and collaborative research ventures over the past decades. We confess that these examples are related most notably to our varied but long-running empirical experiences in the UK, France, and Brazil, and more widely in Latin America, Europe and Southeast Asia. In all of the chapters, however, we also cite the international work of many other rural scholars. We will come back to critically consider and reflect on the progress we have made in developing the eight meta-themes outlined above in the conclusion to the volume.

As we envisage in Figure 0.1, the proceeding eight research agenda chapters are both interlinked by a set of wider transversal dimensions that stem broader 
social science questions, the contribution of rural studies to these, and the ways in which interdisciplinary and transdisciplinary research may proceed. We outline these here at the start of the volume, almost as a set of questions and postulates. They are then taken up in varying degrees in the eight substantive chapters. Then, in conclusion, and as a way of creating some critical and prospective synthesis, we will re-address these cross-cutting themes. We recognize, thus, that our eight-by-eight approach here is not exhaustive of the themes and topics that could be included in such an ambitious agenda. However, we believe that this framing of the rural research agenda does act as a significant and creative 'conceptual and empirical compass', which can direct and stimulate rural researchers and scholars over the coming decades.

So let us now outline the wider transversal themes here by way of concluding this introduction to the volume. These are framed largely as a set of critical questions. There are some that we will return to in the conclusion to the book, while others are embedded in varying degrees in the eight succeeding substantive chapters. They are thus a conceptual framing device in which to embed the substantive chapters and indeed to encourage readers to make links between the chapters (as suggested in Figure 0.1). They are also, more pragmatically, a way of containing the substantially wide and diverse focus on the overall question (see above) that we have set ourselves: what is the current research agenda for rural development studies?

\section{The transversal themes and the framing of meta-research questions}

1. Socio-political dynamics: This concerns the power and politics involved in contestations between hierarchical/distributed control and governance, and the question of the re-democratization and empowerment of power relations over space and through institutions (see Chapter 2 on governance, 3 on power and 5 on land). What are the different power geometries and geographies that are unfolding in rural areas and in urban-rural relations? Will the post-carbon transition lead to just a continuation of centralized systems of control by the public, community and private actors, and public institutions? And/or can we see new experimental associational and institutional arrangements being developed that are emerging in the interstices of established systems of governance and governance practices? Indeed this is inherently a dialectical process, including both conventional forms of domesticated governance and politics (chapters 1 and 2), and wider, more empowering systems of association that re-energize rural 
and urban relations (e.g. in the form of new solidarity movements, new digital-based inter-communication, climate change emergence, extinction movements, etc.). On the other hand, as we shall see in Chapter 2, the rise of counter-nationalist and populist movements, not least drawing upon traditional rural values and property rights, are creating opposite tendencies towards what we term 'disruptive governance'. Are we moving away from a 'post-political' stance in rural research? How do we develop explicit political and ecological integrations?

As many cities and towns cope with combinations of the ecological and neo-liberal crisis that is engulfing them - not least in higher levels of in-migration on continental scales, the social and political reactions to this crisis is to 'blame the other' - often those indeed who have been most dispossessed from the crisis in the first instance. Part of the unintended consequences of the ecological and neo-liberal crisis of reducing resources and choices for many urban residents and dispossessed migrants to cities, is then a political shift towards new authoritarian 'solutions' that express themselves as disruptive forms of governance. We see this in Brazil and the UK and many parts of Europe at the moment. The agri-food and rural sectors become caught up in these geopolitical shifts either directly or indirectly.

2. Socio-economic dynamics: There are concentrated processes of financialization and profit abstraction versus re-territorialized spaces for new and reproduced 'petty capitals', small businesses and profit sharing (see Chapter 4 on financialization). Here we see growing social inequalities leading to new politics and new social movements, and the growth of alternative financing arrangements outwith the dominant financialized frameworks. A key question here is, what are going to be the features of the post-carbonized economy? What degrees of partial, radical decoupling with prevailing industrialized and intensive systems of production and circulation will emerge? And to what extent will rural areas become sites for the 're-coupling' and reconnecting of new, more sustainable forms of (more distributed?) production and circulation? What will be the nature of rural work in this transformation?

3. Socio-natural dynamics: Are new dualisms emerging within nation states rather than just between them? We see the rise of concentrated land and bio-sphere rights and enclosures versus multispecies rights, and 'extinction' movements that stress the re-attribution of new common pool resources and sustainable place making. What moves are there towards new, more nexus-based eco-system matrices of rural relations? There is a potential broadening of the scope of agri-food dynamics to wider nexus and circular economy concerns on the one hand, and linking to wider 
social and urban movements on the other. A deeper understanding of what 'market relations' now mean in this more diversified rural context is necessary (see Chapter 8 on markets and exchange). The role of the social relations underpinning property rights (Chapter 5) is critical here; are they still based upon class lines, or other criteria?

4. Transformational ontologies: The chapters will be contributions to co-produced and pragmatist, multi-perspective approaches to enacting agrarian transformations; and thus of relevance in the practice of rural development research as well as the theory. This involves questions such as: Who are the key actors and assemblages in creating transformations in rural processes and practices? How and by what means are they enacted and mobilized? How much institutional support can and should they expect? How can we build new and adapted rural transformational theory that builds upon earlier transition management models (see chapters 3 and 6)? How do we build the ecological and the bio-physical into these models? How is power assembled and then mobilized? How can knowledge be enacted effectively?

5. Epistemological theories of rural development in post-colonial contexts: We realize the significance of the fact that 'development' is different in different contexts, but that does not deny the significance of real place-based sustainable rural development in its proper and sensitized context. This requires new epistemologies that are sensitized to place-based agency and endogenous and indigenous forms of rural development. What do we mean by sustainable rural development 'in context'; can and should it be generalized or generalizable?

6. Towards pragmatic pathways of rural development at multi-scale levels: How do we bring together more structuralist and more constructivist approaches, given that both are needed in order to foster real sustainable transformations? What examples do we have; is this lacking in the literature?

7. The contested nature of the post-carbon transition, the politics (and economics of denial) versus new sustainable organizations, social innovations and institutional innovations; also the tyranny of short-term thinking and short-term politics - how do we overcome these obstacles; what sorts of political projects do we need to support? How will agro-ecological processes and practices develop in the post-carbon transition (Chapter 6), and what will be the role of transdisciplinary research in progressing these transformations?

8. Finally, there are the social and political dilemmas surrounding new rural development pathways, and in rural society more generally, demographic and consumer-based threats and challenges to the authenticity 
of rural life and society. At the heart of rural development should come understandings of how rural (and urban) people comprehend the dilemmas and contradictions that confront them. Here lies a tension between managing and surviving in their own daily and family/domestic practices, but coping with changes that may for at least a time make them more vulnerable. Why should we expect already vulnerable people to endure the costs of change and transition when it does not seem to be in their short- or medium-term interests? How important are effective forms of community leadership in this regard? Can we expect community leaders to emerge automatically and autonomously, or do they need to be fosters - but by what means can this be achieved? What will be the effects of continued demographic shifts on the politics and economies of rural places? These represent significant and often contradictory dilemmas for rural people and rural researchers. What are the implications for how we go about our research evolving effective co-production methods and practices?

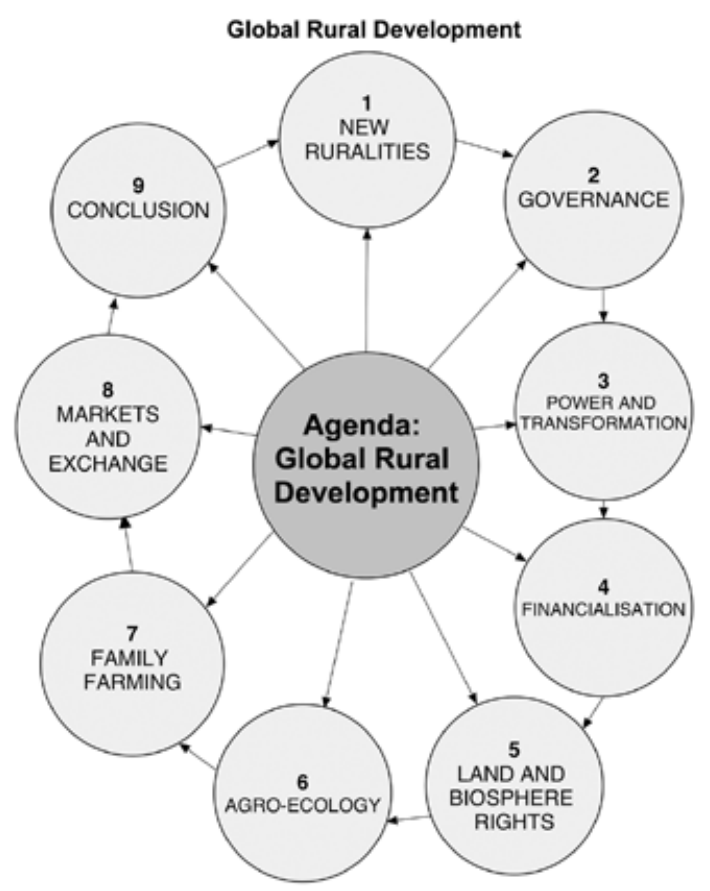

Figure 0.1 Agenda: global rural development flow chart 